\title{
Evaluating Volumetric Brain Registration Performance Using Structural Connectivity Information
}

\author{
Aleksandar Petrović ${ }^{1,2}$ and Lilla Zöllei ${ }^{3}$ \\ ${ }^{1}$ University of Oxford, FMRIB Centre, John Radcliffe Hospital, Headington, \\ Oxford, OX3 9DU, UK \\ ${ }^{2}$ Siemens AG, Healthcare Sector, 91052 Erlangen, Germany \\ ${ }^{3}$ Martinos Center for Biomedical Imaging, Massachusetts General Hospital, \\ Boston, MA 02176, USA \\ petrovic@fmrib.ox.ac.uk, lzollei@nmr.mgh.harvard.edu
}

\begin{abstract}
In this paper, we propose a pipeline for evaluating the performance of brain image registration methods. Our aim is to compare how well the algorithms align subtle functional/anatomical boundaries that are not easily detectable in T1- or T2-weighted magnetic resonance images (MRI). In order to achieve this, we use structural connectivity information derived from diffusionweighted MRI data. We demonstrate the approach by looking into how two competing registration algorithms perform at aligning fine-grained parcellations of subcortical structures. The results show that the proposed evaluation framework can offer new insights into the performance of registration algorithms in brain regions with highly varied structural connectivity profiles.
\end{abstract}

Keywords: Volumetric registration, MRI, probabilistic tractography, structural connectivity, human thalamus.

\section{Introduction}

There have been numerous studies comparing the performance of volumetric registration algorithms by measuring the correspondence between manually outlined labels of the reference and the warped images (e.g. see [1]). These types of evaluations shed light on which algorithm performs better and in which areas. However, they often rely on information that was also used to drive the registration algorithms (e.g. intensity contrast in T1-weighted images). In order to avoid such circularity, ideally, an independent testing mechanism should be used. In other words, one should avoid the circularity of the information flow as well as 'using registration to assess registration'. This paper tackles the former issues and tries to minimize the influence of the latter.

Our paper proposes a new framework for evaluating performance differences of volumetric registration algorithms. It relies on a modality different from the one used for registration (in our case, T1-weighted images). Diffusion Weighted Imaging (DWI) is a suitable choice for this purpose as it contains information complementary to that found in other structural modalities. In this paper we assess the inter-subject alignment of anatomical boundaries within subcortical structures derived through the 
analysis of white matter connectivity. Note, the aim of this paper is to present an evaluation methodology and not to provide a comprehensive comparison of a range of registration methods.

DWI has been used to map the architecture of cortical white matter (WM) and also to determine WM consistency [2,3,4]. Furthermore, WM connectivity patterns highly correlate with the functional segregation of the brain [5]. Thus, evaluating registration performance in the areas of distinct connectivity patterns might yield additional insights into the inter-subject alignment of functional homologues.

We were inspired by the work of [6] which shows that thalamic connectivity analysis can result in anatomically and functionally plausible and highly consistent parcellations of the anatomy, revealing structures (i.e. different thalamic nuclei) that cannot be seen on T1- or T2-weighted images alone. Therefore, if we were to: 1) parcellate all the thalami in a group of subjects, e.g. as proposed in [7], and 2) consistently label anatomically corresponding clusters across subjects, it would be possible to measure inter-subject cluster overlap. Importantly, the registration algorithms assessed by this measure do not use information used for parcellation. The thalamus and the putamen were used as examples to demonstrate our findings.

\section{Methods}

In order to demonstrate our hypothesis, this paper evaluates the differences between the Combined Volumetric and Surface-based registration (CVS) algorithm [8, 9] and FNIRT (FMRIB's non-linear image registration tool) [10] by examining the postregistration overlap of connectivity-based segmentation labels across a group of control subjects. CVS and FNIRT belong to two complementary and widely used software packages and it is of great interest to the neuroimaging community to know which registration method of these two is superior in what case.

First, for each subject, image distortion and bias field corrections were performed prior to the DW-to-T1 affine registration (FSL/FLIRT [13]). In that way, pairs of DW and T1 images were brought to the same coordinate system prior to further analysis. Secondly, in order to perform parcellation of subcortical structures using structural connectivity, every voxel in the region of interest (ROI, e.g. the thalamus) has to be associated with a feature vector encoding measures of connectivity to other brain areas. These feature vectors become inputs into the clustering algorithm. Commonly, each entry in the feature vector is the probabilistic tractography result (a measure of uncertainly of the WM tract originating in the seed voxel and passing through the target) to one of the neocortical targets, e.g. a major brain lobe [6]. In order to boost the sensitivity in discriminating between areas of varying connectivity, we decided to identify a greater number of cortical targets using the spherical registration and parcellation algorithm of FreeSurfer [12]. Targets for probabilistic tractography (PROBTRACKX/FSL [13]) were formed in the following steps:

1) Using Freesurfer, parcellate surfaces of both cortical hemispheres into 80 equilateral triangles (calculated as the first subdivision of an icosahedron) that are consistently labeled across all subjects. Triangular features were chosen as they do not follow any particular anatomical divisions and do not therefore bias the results; 

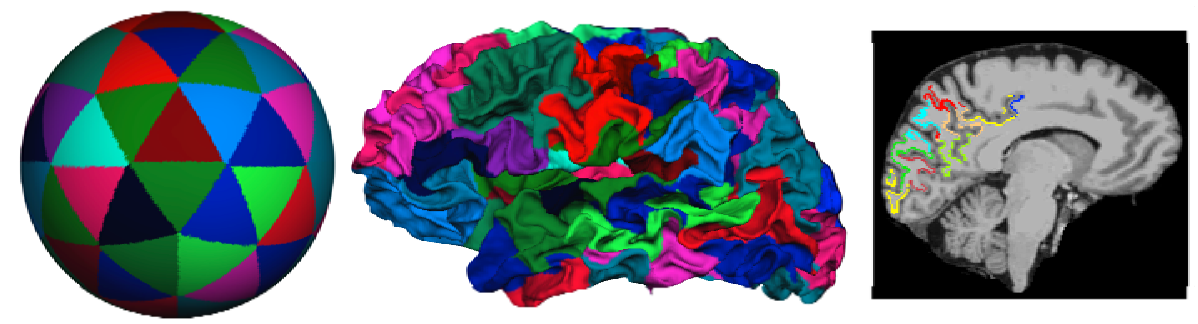

Fig. 1. Triangular targets were drawn on an inflated and registered sphere (left) thus imposing anatomical consistency in triangle positions across subjects. Figures (middle and right) show projections of triangles to the white matter surface - different colours correspond to different triangle labels, i.e. different cortical targets.

2) Propagate surface parcellations into the volume. A $1 \mathrm{~mm}$ thick sheath on the grey/white matter surface was constructed as depicted in 1) (see Fig. 1);

3) Concatenate volumetric masks/triangular labels from both hemispheres to form a list of cortical targets (targets for probabilistic tractography).

Therefore, the final output of probabilistic tractography for every ROI is an MxN connectivity matrix where $\mathrm{M}$ is the number of voxels in the ROI and $\mathrm{N}$ is the number of targets, which in our current implementation is set to 160 (80 targets for each hemisphere). Typically, connectivity matrices are very sparse having $90-95 \%$ of all values zero. Clustering of the thalamic and putamen connectivity matrices is done for all 41 subjects using Hierarchical Dirichlet Process Mixture Models (HDPM) with spatial constraints [7]. Both structures were segmented by FreeSurfer [11]. Each of the connectivity matrices was preprocessed and grouped into 9 clusters. Preprocessing consisted of PCA data dimensionality reduction to size Mx20, typically retaining more than $95 \%$ of explained variance. We decided not to use infinite mixture models in our analysis as they regularly produced more than 40 clusters, many of which contained just a few voxels. By varying the number of clusters for a small subset of subjects, we found that 9 clusters is a good compromise between capturing anatomically plausible divisions and achieving reasonable inter-subject consistency. Clustering consistency was also found to be robust with respect to the number of cortical targets. The parameter regulating intra-subject spatial smoothness (called 'beta', as implemented in [7]) was set to 1, which is the default value suggested in [7]. We should note that HDPM-based clustering does not make use of inter-subject registrations and the correspondence among cluster labels is established solely according to the data matrix fed into the HDPM pipeline.

After the clustering step, all subjects were non-linearly registered to a template (which was part of the hierarchical clustering cohort, a randomly chosen subject) using two methods: CVS and FNIRT (see Sections 2.1 and 2.2). We decided not to use a standard space (such as the MNI152) for our analysis, as we test for the alignment of very subtle and subject-specific parcellations and are thus in need for a template image with similar detail in it. We believe that if the T1-weighted intensities within our ROIs are insufficient to discriminate between internal subregions, then the 
alignment of other cortical areas would constrain the deformations, hopefully maximizing the alignment of functionally distinct areas.

Finally all clustering results were warped using the two registration methods. We then computed the Jaccard overlap measure to test the inter-subject alignment accuracy among the HDPM clustering of the connectivity descriptors in the ROIs.

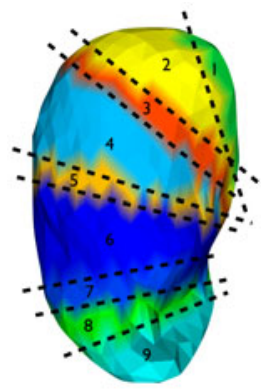

Thalamus, ventral view

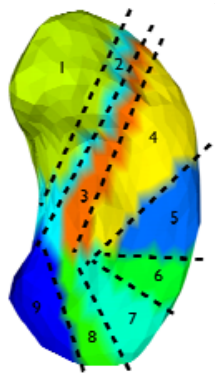

Putamen, medial ventral view

Fig. 2. Thalamus and Putamen clustered using proposed methodology (HDPM clustering of connectivity matrices). Different colours/numbers represent different clusters ( 9 in total) of distinct WM connectivity patterns, e.g. thalamic nuclei (image left).

\subsection{CVS}

Combined Volumetric and Surface-based Registration is a brain image registration method that maximizes the alignment of both cortical and subcortical structures. It consists of three image processing steps. First, a surface registration algorithm finds correspondences between the input surfaces from two brain scans [14] and these correspondences are transformed into a sparse displacement field in Euclidean space. This morph is then diffused into a dense displacement field in the volume using a nonlinear elastic model. Finally, a nonlinear volumetric registration refines the alignment, bringing subcortical structures, which are not near the surfaces, into accurate alignment. This technique has been shown to produce state-of-the-art alignment of cortical folding patterns, architectonics and subcortical structures [8].

\subsection{FNIRT}

The nonlinear FNIRT registration tool [10] uses a B-splines representation of the registration warp field and optimizes the sum of squared differences as its objective function. It is not typically run in a subject-to-subject setting as it was initially optimized for subject-to-template registrations. For our project, together with the original developers of the code, we established a particular set of parameters that achieve high quality subject-to-subject correspondence.

\subsection{Data Description}

The experiments were run on data provided through collaboration with Dr. Randy Gollub and the Medical Investigation of Neurodevelopmental Disorders (MIND) Institute. Forty-one data sets were selected all of which have been acquired by our 
collaborators using an identical MRI sequence on a Siemens scanner. The structural data is of $256 \times 256 \times 256$ size with $1 \mathrm{~mm}^{3}$ voxel resolution and $\mathrm{TR}=12 \mathrm{~ms}$, TE $=4.76 \mathrm{~ms}$, $\mathrm{TI}=4.76 \mathrm{~ms}$, flip angle $=20^{\circ}$. The diffusion scans use single shot EPI, and a twicerefocused SE pulse sequence, optimized to minimize eddy current-induced distortions $\left(\mathrm{TR} / \mathrm{TE}=7400 / 89 \mathrm{~ms}, \mathrm{~b}=700 \mathrm{~s} / \mathrm{mm}^{2}, 256 \times 256 \mathrm{~mm}\right.$ FOV, 128x128 matrix, $2 \mathrm{~mm}(0$ mm gap) slice thickness, 10 T2 + 60 DWI. Sixty-four slices were acquired in the ACPC plane. The 60 diffusion-encoding gradient directions were determined using the electrostatic shell method, and result in a high signal-to-noise diffusion volume.

\section{Results}

Comparison between the FNIRT and CVS registration methods was performed for thalamic and putamen clusterings. Table 1 summarizes the results of compound (calculated across all labels in parallel) Jaccard coefficient measures. Using this measure every subject's clustering was compared to the template clustering. In summary, FNIRT performs consistently better than CVS in the left thalamus.

Table 1. Mean Jaccard coefficient between the clustering of the template and all the other thalami/putamen (40 comparisons per table entry - see Fig. 3). Statistically significant difference at $\mathrm{p}<0.01$ level is indicated in bold (unpaired T-test, DOF correction).

\begin{tabular}{lll||lll}
\hline THALAMUS & FNIRT & CVS & PUTAMEN & FNIRT & CVS \\
\hline Left & $\mathbf{0 . 1 9}$ & 0.14 & Left & 0.20 & 0.18 \\
Right & 0.16 & 0.13 & Right & 0.21 & 0.20 \\
\hline
\end{tabular}

We also calculated how well both methods perform when each ROI is considered a single label, without any clustering. In both the left and right hemispheres, FNIRT performed better $(\mathrm{p}<0.001)$ over CVS. If this effect is regressed out of the findings from Table 1, previous conclusions do not change (FNIRT performs better in the left thalamus with $\mathrm{p}<0.05$ ). Regressing out an effect, in this case, means calculating the compound Jaccard coefficient just for the intersection of the two ROIs.

We also calculated the pairwise compound Jaccard coefficient (compound Jaccard coefficient calculated between each pair of subjects) for the whole dataset excluding the template. These results are shown in Table 2. Similarly to the previous analysis, we computed this metric with the ROIs treated as a single label. In both the left and right hemispheres, FNIRT performed better $\left(\mathrm{p}<10^{-5}\right)$. When the effect of the global alignment is regressed out of the findings from Table 2, FNIRT still outperformed CVS for both the left and right putamen and for the left thalamus $(\mathrm{p}<0.01)$.

Table 2. Mean pairwise compound Jaccard coefficient (see [8] for computational details) among the clusterings of all ROIs excluding the template (40x40 comparisons per table entry see Fig. 3). Statistically significant difference at $\mathrm{p}<0.01$ level is given in bold (unpaired T-test, DOF correction).

\begin{tabular}{lll||lll}
\hline THALAMUS & FNIRT & CVS & PUTAMEN & FNIRT & CVS \\
\hline Left & $\mathbf{0 . 2 8}$ & 0.23 & Left & $\mathbf{0 . 2 6}$ & 0.22 \\
Right & $\mathbf{0 . 2 7}$ & 0.20 & Right & $\mathbf{0 . 1 8}$ & 0.15 \\
\hline
\end{tabular}



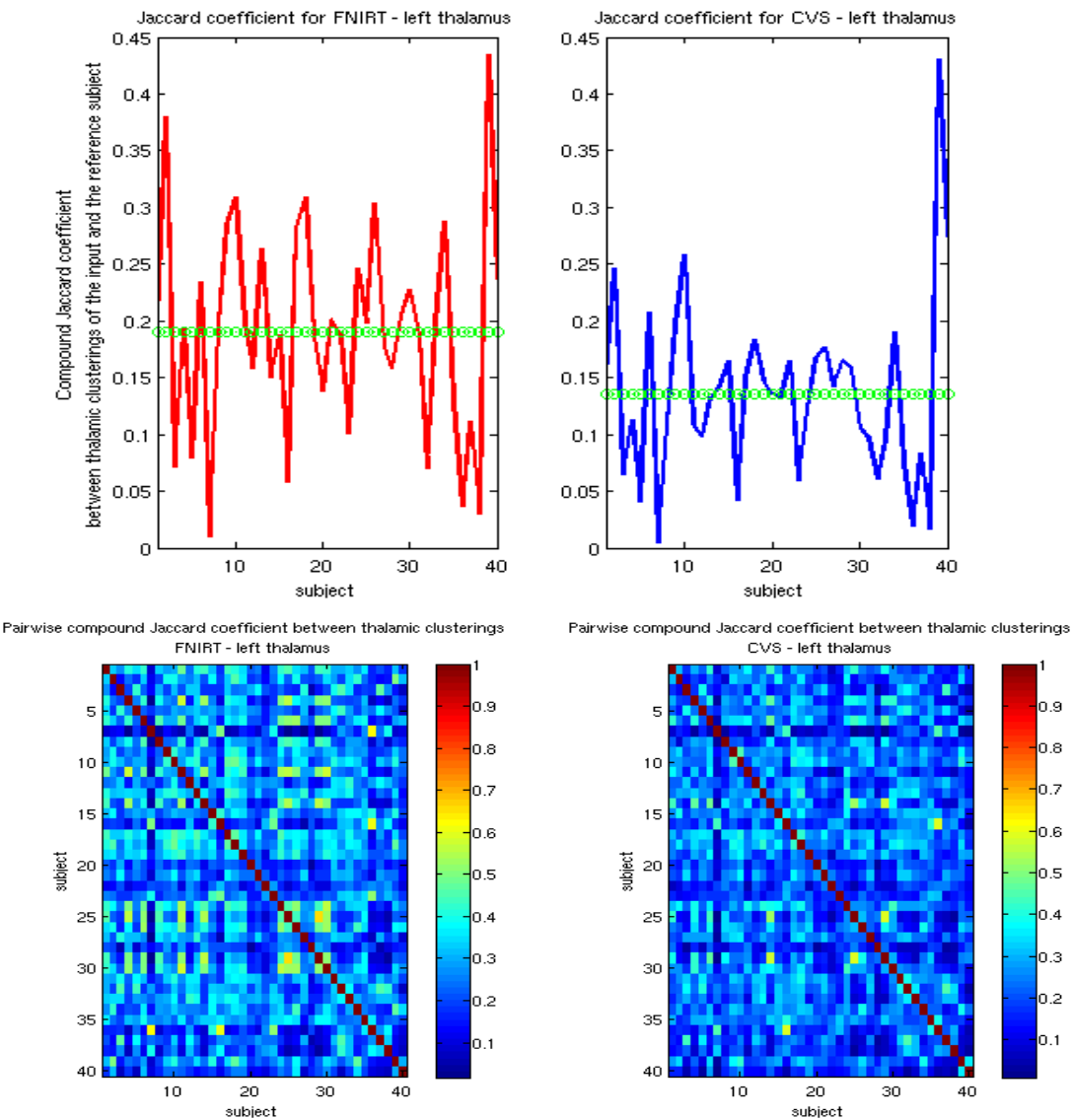

Fig. 3. (FIRST ROW) Compound Jaccard coefficient for the left thalamus for FNIRT and CVS algorithms. Mean Jaccard coefficient value is shown with a green line (see Table 1). (SECOND ROW) Pairwise compound Jaccard coefficient for the clusterings of the left thalamus for FNIRT and CVS algorithms (see Table 2). Subjects are numbered from 1 to 40.

\section{Discussion}

A new method for evaluating registration performance is proposed. It tests for registration differences within areas of uniform T1/T2 contrast, but of highly variable connectivity (such as the thalamus and the putamen).

The results show that it is possible to make highly local distinctions between competing registration methods possibly giving a new insight into how and where they can be improved. However, our pipeline so far includes automatically segmented subcortical labels, which is suboptimal to having expert segmentations. The questions such as what are the best ways to cluster connectivity matrices, impose inter-subject cluster correspondences and how these affect final findings also need further investigation. Furthermore, we would like the registration methods (that are 
compared) to be independent of the evaluation pipeline. In this particular demonstration, CVS relies on FreeSufer cortical surface registrations which might introduce a potential confound (possibly in favor of CVS).

Although our goal is to compare performance of volumetric registration methods by looking into the overlap of anatomical boundaries found through WM connectivity analysis, we acknowledge that the accuracy of registration itself can be application specific [15]. E.g., this particular evaluation pipeline can yield an "optimal" registration method to be used when analyzing the group results of a functional paradigm with activations in deep brain structures. A "better" registration method could, in that case, result in statistically stronger conclusions and/or better spatial localization of the measured effect.

Finally, we hope to motivate further research into the integration of connectivity information (both structural and functional) into registration algorithms. E.g., the structural connectivity matrix utilized in this paper (and comparable across subjects) can be directly integrated into the registration cost functions of CVS or FNIRT [16].

Acknowledgments. This work was supported by the Oxford University Full Clarendon Award and NIH grant 1K99HD061485-01A1. Authors would like to thank Dr. Mark Jenkinson and Prof. Bruce Fischl for useful comments and advice.

\section{References}

1. Klein, A., Ghosh, S.S., Avants, B., Yeo, B.T.T., Fischl, B., Ardekani, B., Gee, J.C., Mann, J.J., Parsey, R.V.: Evaluation of volume-based and surface-based brain image registration methods. Neuroimage 51, 214-220 (2010)

2. Smith, S.M., Jenkinson, M., Johansen-Berg, H., Rueckert, D., Nichols, T.E., Mackay, C.E., Watkins, K.E., Ciccarelli, O., Cader, M.Z., Matthews, P.M., Behrens, T.E.: Tractbased spatial statistics: voxelwise analysis of multi-subject diffusion data. Neuroimage 31, 1487-1505 (2006)

3. Behrens, T.E., Berg, H.J., Jbabdi, S., Rushworth, M.F., Woolrich, M.W.: Probabilistic diffusion tractography with multiple fibre orientations: What can we gain? Neuroimage 34, 144-155 (2007)

4. Jbabdi, S., Woolrich, M.W., Andersson, J.L., Behrens, T.E.: A Bayesian framework for global tractography. Neuroimage 37, 116-129 (2007)

5. Klein, J.C., Behrens, T.E., Robson, M.D., Mackay, C.E., Higham, D.J., Johansen-Berg, H.: Connectivity-based parcellation of human cortex using diffusion MRI: Establishing reproducibility, validity and observer independence in BA 44/45 and SMA/pre-SMA. Neuroimage 34, 204-211 (2007)

6. Johansen-Berg, H., Behrens, T.E., Sillery, E., Ciccarelli, O., Thompson, A.J., Smith, S.M., Matthews, P.M.: Functional-anatomical validation and individual variation of diffusion tractography-based segmentation of the human thalamus. Cereb Cortex 15, 31-39 (2005)

7. Jbabdi, S., Woolrich, M.W., Behrens, T.E.: Multiple-subjects connectivity-based parcellation using hierarchical Dirichlet process mixture models. Neuroimage 44, 373-384 (2009)

8. Postelnicu, G., Zöllei, L., Fischl, B.: Combined volumetric and surface registration. IEEE Trans. Med. Imaging 28, 508-522 (2009) 
9. Zöllei, L., Stevens, A., Huber, K., Kakunoori, S., Fischl, B.: Improved tractography alignment using combined volumetric and surface registration. Neuroimage 51, 206-213 (2010)

10. Andersson, J., Jenkinson, M., Smith, S.: Non-linear registration, aka spatial normalisation. Oxford Centre for Functional Magnetic Resonance Imaging of the Brain, Department of Clinical Neurology, Oxford University, Oxford, UK (2007)

11. Fischl, B., Salat, D.H., Busa, E., Albert, M., Dieterich, M., Haselgrove, C., van der Kouwe, A., Killiany, R., Kennedy, D., Klaveness, S., Montillo, A., Makris, N., Rosen, B., Dale, A.M.: Whole brain segmentation: automated labeling of neuroanatomical structures in the human brain. Neuron 33, 341-355 (2002)

12. Fischl, B., Liu, A., Dale, A.M.: Automated manifold surgery: constructing geometrically accurate and topologically correct models of the human cerebral cortex. IEEE Trans. Med. Imaging 20, 70-80 (2001)

13. Woolrich, M.W., Jbabdi, S., Patenaude, B., Chappell, M., Makni, S., Behrens, T., Beckmann, C., Jenkinson, M., Smith, S.M.: Bayesian analysis of neuroimaging data in FSL. Neuroimage 45, S173-S186 (2009)

14. Fischl, B., Sereno, M.I., Tootell, R.B., Dale, A.M.: High-resolution intersubject averaging and a coordinate system for the cortical surface. Hum. Brain Mapp. 8, 272-284 (1999)

15. Yeo, B.T., Sabuncu, M., Golland, P., Fischl, B.: Task-optimal registration cost functions. Med. Image Comput. Comput. Assist. Interv. 12, 598-606 (2009)

16. Petrović, A.: Connectivity Driven Registration of Magnetic Resonance Images of the Human Brain. DPhil Thesis University of Oxford (2011) 